\title{
CORRELATION STUDY OF PLANETARY-BOUNDARY-LAYER- HEIGHT RETRIEVALS FROM CL51 AND CHM15K CEILOMETERS WITH APPLICATION TO PM2.5 DYNAMICS IN NEW YORK CITY Dingdong $\mathrm{Li}^{1}$, Barry Gross ${ }^{1,2}$, Yonghua $\mathrm{Wu}^{1,2}$, Fred Moshary ${ }^{1,2 * *}$ \\ ${ }^{1}$ Optical Remote Sensing Lab, City College of New York, USA \\ ${ }^{2}$ NOAA - Cooperative Science Center for Earth System Sciences and Remote Sensing Technologies Email:moshary@ccny.cuny.edu
}

\begin{abstract}
Planetary-boundary-layer-height (PBLH) plays a critical role in the study of urban air quality, weather and climate. Continuous observation is critical in understanding air pollution processes and evaluation of air quality/ weather models in the complex urban environment. In this study, we observe the PBLH variation using multiple ceilometers and lidar in New York City (NYC) during both the summer and winter time and explore the potential correlation with ground PM2.5. An automated quality control and quality assurance (QC/QA) method is developed to optimize the PBLH determination from the ceilometers (Vaisala CL51 and Lufft CHM15k) product. The PBLHs from the two ceilometers and lidar show good consistency $\left(\mathrm{R}^{2}=0.68 \sim 0.88\right)$ during the convective PBL period at 15:00-21:00 UTC (10:00-16:00 EST). We also investigate the seasonal variation and diurnal evolution of PBLH and demonstrate an inverse relation between the PBLH and PM2.5 during the morning transient period of PBLH growth. Further, the correlation between the ceilometer-attenuated backscatter and ground PM2.5 and its dependences on the vertical altitude are analyzed, showing that the aerosols in the PBL are more deeply mixed while also being influenced by the relatively high humidity variability during the summer.
\end{abstract}

\section{INTRODUCTION}

In conventional air quality monitoring of fine particulate matter (PM2.5) performed by the EPA, observations are generally limited to the surface level [1]. Although such surface monitoring is critical due to specific EPA NAAQS health standards for PM2.5 exposure [2], surface measurements alone do not provide insight into how ambient aerosols mix within the planetary boundary layer. In particular, diurnal cycle and season variation can have a dramatic effect on both the mixing height and the homogeneity of the aerosols. These mechanisms drive the venting of aerosol emission sources and allow pollution to vent through the PBL, resulting in a basic anticorrelation between PBLH and the surface pollution under well mixed conditions.

In prior researches at $\mathrm{CCNY}, \mathrm{Nd}$ :YAG high power lidar was employed to extract PBLH information using WRF models [3]. Despite of high SNR, such lidar system can only measure a limited set of days due to manual operation and eye safety concerns and therefore is not suitable for long-term continuous studies. In this case, automatic lidar and ceilometer (ALC) instruments are advantageous for urban atmosphere observation given their continuous 24-hour measuring capacity of atmospheric profiling including cloud base height, aerosol attenuated backscatter coefficient and PBLH. In previous WRF-CMAQ PBLH and PM2.5 analyses [3], a Vaisala CL-31 ceilometer was used to retrieve the PBLH. However, the relatively weaker laser power of CL-31 leads to a lower signal-noise-ratio (SNR). And the lower SNR makes it more difficult to resolve PBLH $>1$ $\mathrm{km}$ and find unique aerosol layer structure within the PBL.

To improve observational capacity, more powerful and robust ceilometers with higher SNR are desired for PBL and aerosol profile dynamics study. Compared to Vaisala CL-31, Vaisala CL51 and Lufft CHM15k have better SNR, higher detection range, but at the same time, suffer from longer optical non-overlap length. Under clear sky condition and in strong convective PBL period, the aerosols are well mixed, in which circumstance the PBLH often refers to mixing-layer-height (MLH), the PBLH results from different ALCs are highly consistent. But complex atmospheric conditions such as multiple aerosol layers, clouds or rainy weather will significantly affect ceilometer PBLH detection accuracy. Therefore, quality assurance/ 
quality control (QA/QC) post-process is necessary to exclude extraneous layer heights, outliers and data of rainy weather and thus improves the PBLH product quality and its applications in the air quality assessment.

\section{METHODOLOGY}

\subsection{Observation instruments}

In comparison study, the attenuated backscatters and PBLHs are retrieved by a Vaisala CL51 ceilometer, a Lufft CHM15k ceilometer and a 3wavelength Elastic-Raman (Nd:YAG) lidar coupled with LICEL transient recorders (TR40160) deployed on the roof of the Engineering School of City College of New York (CCNY). The distance between these three instruments is $5-10 \mathrm{~m}$ to avoid signal interference but close enough to collect data from the same atmospheric volume. Nearby is a standard surface air quality monitoring station by the New York State Department of Environment Conservation (NYSDE), collecting air quality data (PM2.5, ozone and carbon monoxide concentration) with 1-hour collection time. The Vaisala CL51 ceilometer operates at a wavelength of $910 \pm 10 \mathrm{~nm}$ at $25^{\circ} \mathrm{C}$, and the measurement range is $0-10,000 \mathrm{~m}$. The time/height resolution of the CL51 ceilometer is set to be $16 \mathrm{~s}$ $/ 10 \mathrm{~m}$. The center wavelength of Lufft CHM15k is $1064 \mathrm{~nm}$ and the measurement range is $5-15,000$ $\mathrm{m}$, with time/height resolution of $15 \mathrm{~s} / 15 \mathrm{~m}$, respectively. The lidar transmits three wavelengths (1064-, 532- and 355-nm) with a flash lamppumped Nd: YAG laser at a repetition rate of $30 \mathrm{~Hz}$ (Spectra-physics Quanta-Ray PRO-320). The two ceilometers make automatic 24-hour observation in all-weather conditions, while the lidar requires manual operation and thus only collects data in the daytime under appropriate weather condition. All data recording is based on UTC and the data analysis is done in MATLAB R2018b.

\subsection{Data processing}

To improve the SNR and align the timestamps of the data from two ceilometers, the attenuated backscatters of CL51 and CHM15k are averaged every 10 minutes. Along the range dimension, a $150 \mathrm{~m}$ sliding window is applied to the backscatters and the maximum range is set to be $4500 \mathrm{~m}$ which captures all PBL aerosols but excludes high clouds and smoke plumes. Additionally, in PM2.5 and backscatter correlation evaluation (section 3.3), the attenuated backscatter coefficient of lower clouds is excluded based on the histogram of attenuated backscatter from that day.

Both ceilometers retrieve PBLH using negative gradient of the backscatter profile and provide up to three levels of PBLH attributions of each time. Here, the lowest detectable PBLH attribution is set to be the primary PBLH. To reduce the calculation consumption, the PBLHs are averaged every 10 minutes. Afterwards, a $\mathrm{QA} / \mathrm{QC}$ post-process is implemented as follows:

- Initiate PBLH false positive flags (pbltfp) as 0 for each PBLH;

- Compare the PBLHs before sunrise $\left(\mathrm{PBLH}_{\mathrm{bfs}}\right)$, morning transition $\left(\mathrm{PBLH}_{\mathrm{mt}}\right)$, afternoon $\left(\mathrm{PBLH}_{\mathrm{aftn}}\right)$ with thresholds $\mathrm{T}_{1}, \mathrm{~T}_{2}$, $\mathrm{T}_{3}$, respectively, if PBLH greater than the corresponsing threshold, then pbltfp $=1$;

- Compare the PBLHs after sunset (PBLH $\left.{ }_{\text {afst }}\right)$ with PBLH in the noon time, if $\mathrm{PBLH}_{\mathrm{afst}}>$ $\mathrm{PBLH}_{\text {noon, }}$, then pbltfp=1;

- Find the shape peak/valley of PBLHs of an entire day using MATLAB built-in function 'findpeaks' (Copyright 2007-2016 The MathWorks, Inc.) and set their pbltfp $=1$;

- If PBLH > mean (PBLHs) \pm 3 std, pbltfp $=1$;

Apart from our QA/QC process of PBLH, the sky condition is evaluated by duration time of near surface backscatters which are greater than the threshold, indicating rain, fog or dust on the lens. If such duration time is more than 3 hours, the data file of this day will not be considered.

\section{RESULTS and DISCUSSION}

\subsection{MLH correlation of lidar \& ceilometers}

Figure 1 shows the correlation of the MLHs from lidar with MLHs from CHM15k (Figure 1 (a)) and from CL51 (Figure 1 (b)). The MLHs are collected in 10:00-17:00 (EST) of 7 clear sky days (no rain). Generally, MLHs from both ceilometers are strongly correlated with MLHs from lidar measurement. But the CHM15k leads to a more accurate retrieval $\left(\mathrm{R}^{2}=0.81\right)$ with less root mean square error $(\mathrm{RMSE}=0.154)$. MLHs from CL51 are less consistent with lidar measurement $\left(\mathrm{R}^{2}=0.68\right.$, RMSE $=0.204$ ), which is mainly caused by lower SNR of CL51. 


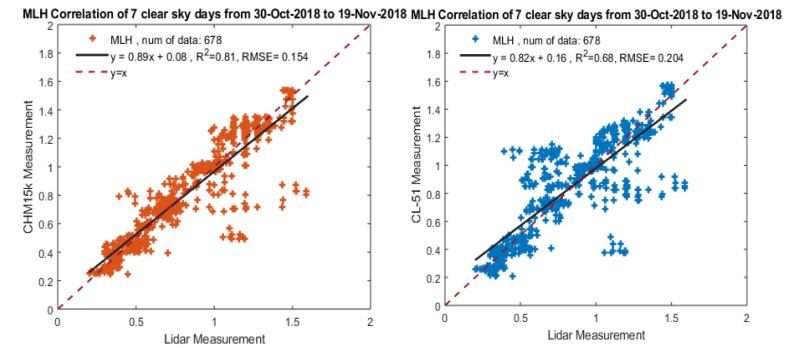

Figure 1. MLHs comparisons between lidar and ceilometers (a) CHM15k (b) CL51

\subsection{PBLH correlation between the ceilometers}

We calculated the correlation coefficient of PBLHs retrieved by both ceilometers for 24 hours over 17 clear sky days (no rain) in November and December 2018. Before QA/QC process, the correlation of PBLHs from both ceilometers is very poor with $\mathrm{R}^{2}=0.1 \sim 0.2$ (not shown). After QA/QC process, the correlation between PBLHs from two ceilometers improves to $\mathrm{R}^{2}=0.76$ with $\mathrm{RMSE}=0.172$. Figure 2 shows the correlation coefficient $\mathrm{R}^{2}$ of PBLHs from two ceilometers in different time periods. Strong correlation appears in 10:00-16:00 (EST) which is the convective PBL period. However, the correlation degrades in the nocturnal period.

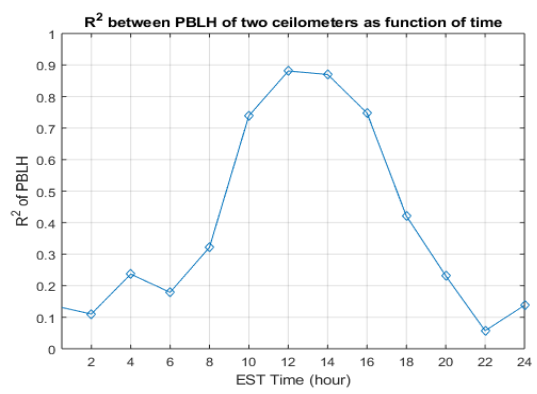

Figure 2. PBLH comparisons between CHM15k and CL51

Such discrepancy can be possibly explained by the following effects: 1 . The influence from the residual layers. 2. The nocturnal PBLH during the fall/winter can be below the non-overlap range of CHM15k, so CHM15k detects higher aerosol layers. 3. The aerosol concentration is too low for ceilometers to capture the PBLH.

\subsection{PBLH potential impact on PM2.5}

With the PBLHs obtained from both ceilometers (no data from CHM15k during the summer), the averaged diurnal PBLHs in July, August and
November, December 2018 under the clear sky condition (no rain) are calculated, in comparison with the averaged diurnal PM2.5 concentration of the same days. The hourly PM2.5 data are from air quality station in CCNY operated by NYSDEC.

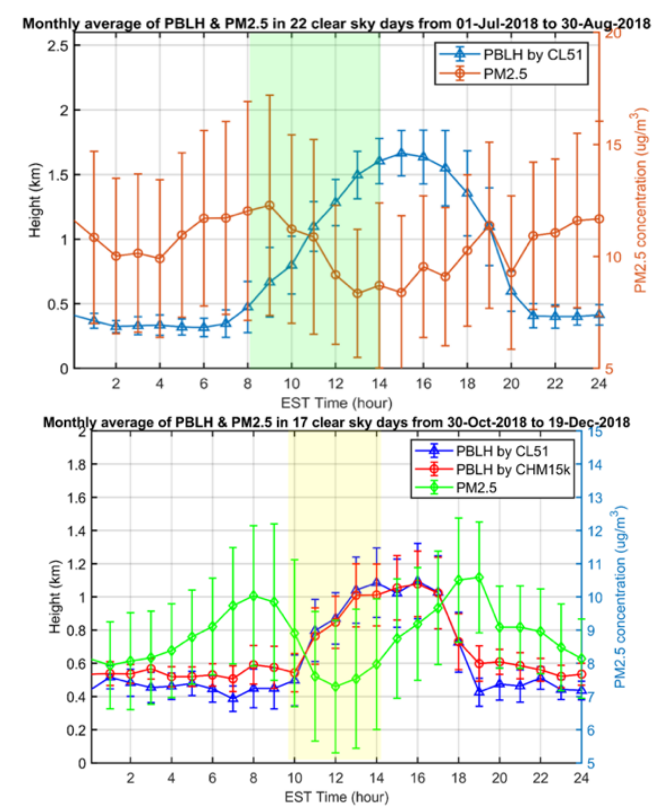

Figure 3. Inverse relationship exhibited between surface PM2.5 and PBL height during morning transition. (a) The diurnal averaged PBLHs measured by CL51 (no CHM15k data during summer) and the diurnal averaged PM2.5 in summer. (b) The diurnal averaged PBLHs measured by CL51 and CHM15k compared with the diurnal averaged PM2.5 in fall/winter.

In Figure 3 (a), the averaged diurnal PBLH demonstrates that PBLH starts to increase around 8:00 and reaches the maximum at $1670 \mathrm{~m}$ around 15:00 with growth rate $\sim 174 \mathrm{~m} /$ hour. Meanwhile, PM2.5 reaches the peak around 9:00 and starts to decrease as the PBLH increases, then fluctuates but slightly increase in the rest time. Figure 3 (b) shows the averaged diurnal PBLHs calculated from CL51 measurements (blue line) and from CHM15k measurements (red line). Here we can see that the averaged PBLH from CHM15k is slightly higher than PBLH from CL51 during the nocturnal period, inferring that CHM15k tends to detect higher aerosol layer heights during the night time as mentioned before. In fall/winter, the morning transition of PBLH starts from 10:00, and PBLHs reach the peak at $1100 \mathrm{~m}$ around 2:00 (growth rate $147 \mathrm{~m} /$ hour). The PM2.5 diurnal cycle in 
fall/winter is similar to the summer, showing two peaks around 8:00 and 18:00 which is probably caused by car emission. Strong inverse relation between PBLH and PM2.5 also appears in the morning transition period, but slightly shifted forward compared to the result in summer. This inverse relation between PBLH and PM2.5 implies that the aerosol particles are diluted when the PBLH growth leads to larger volume of PBL.

\subsection{Correlation of backscatter \& PM2.5}

Next, we explore the correlation coefficient of the attenuated backscatter at different heights with the surface level PM2.5 under clear sky condition. In particular, the result illustrates if the attenuated backscatter can be used as a proxy for fine particle matter and provides a better understanding of the mixing effect of PBL on the pollutants at surface level and upper atmosphere. Figure 4 left panel displays the correlation dependence on vertical altitude for both ceilometers in winter. The CHM15k correlation degradation below $100 \mathrm{~m}$ is due to its larger non-overlap range. The correlation between PM2.5 and PBLHs by CL51 in summer and fall/winter are shown in figure 4 right panel. Note that near field correlation is slightly better in winter which is probably due to the smaller changes of relative humidity in winter affecting the mass to optical coefficient relationship. Besides, the correlation decreasing rate with respect to the height is smaller for summer than that of the winter, implying the deeper mixing effect of aerosols during the summer.
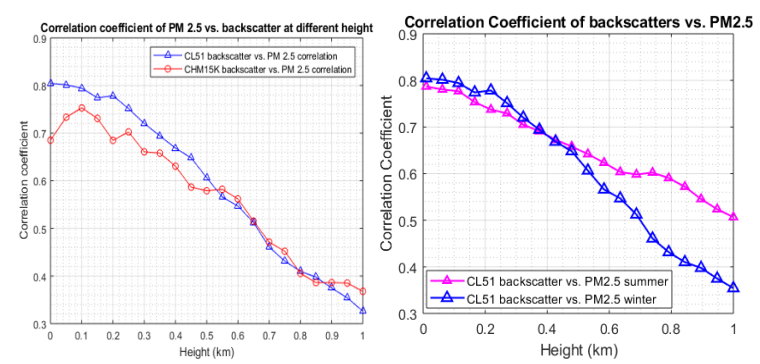

Figure 4. Left: Correlation as function of height between backscatter from two ceilometers and surface PM2.5 concentrations. Right: Correlation as function of height between backscatter from CL51 and surface PM2.5 concentrations in winter and summer.

\section{CONCLUSIONS}

This study demonstrates the potential of using ceilometers for PBL dynamics studies in complex urban environments. We compared and analyzed the PBLH results retrieved from lidar, CL51 and CHM15k ceilometers and optimized by an automated QA/QC method. Diurnal averaged PBLH were compared with PM2.5 during summer and fall/winter, showing strong inverse relation between them during the morning transition period. Finally, backscatter and PM2.5 exhibits strong correlation showing that the ALC measured attenuated backscatter has potential to serve as a proxy for particle mass, but the conversion coefficient of attenuated backscatters to PM2.5 need to be further addressed. In the future study, more climatic factors and larger date set should be assessed to fully understand the mechanism behind the PBLH and air quality and to assess PBL retrievals in Meteorological Models.

\section{ACKNOWLEDGEMENTS.}

This work is supported by the New York State Energy Research and Development Authority under Grant No.100415 and No.137482, and the he National Oceanic and Atmospheric Administration - Cooperative Science Center for Earth System Sciences and Remote Sensing Technologies under the Cooperative Agreement Grant \#: NA16SEC4810008. We appreciate the data from NYS Department of Environmental Conservation. The statements contained within the manuscript are not the views of the funding agency or the U.S. government, but reflect the authors' views.

\section{REFERENCES}

[1] https://www3.epa.gov/ttn/amtic/contmont.html [2] https://www.epa.gov/criteria-air-pollutants/naaqstable

[3] C.-M. Gan, Y. Wu, B.L. Madhavan, B.Gross, F. Moshary, Application of active optical sensors to probe the vertical structure of the urban boundary layer and assess anomalies in air quality model PM2.5 forecasts, Atm. Environment 45, 6613-6621 (2012)

[4] Wu, Y., S. Chaw, B. Gross, et al., 2009: Low and optically thin cloud measurements using a Raman-Mie lidar, Appl. Opt., 48, 1218-1227. 